\title{
The method of fundamental solutions for three-dimensional scattering of elastic waves in layered half space
}

\author{
Zhongxian Liu ${ }^{1}$, Jianwen Liang ${ }^{2}$ \& Zhenning $\mathrm{Ba}^{2}$ \\ ${ }^{1}$ Key Laboratory of Soft Soils and Engineering Environmental of \\ Tian jin Province, Tianjin Chengjian University, China \\ ${ }^{2}$ Department of Civil Engineering, Tianjin University, China
}

\begin{abstract}
This paper presents a new method of fundamental solution (MFS) for solving the scattering of elastic waves in layered half space. Based on dynamic Green's function in layered half space, the proposed method can efficiently and accurately solve three-dimensional wave motion in a layered medium. Numerical results indicate that the amplification effects of elastic waves in a layered basin is more significant than that in a homogeneous basin, thus it is necessary to consider the bedding structure of the alluvial basin for seismic wave modelling in reality.

Keywords: scattering of elastic waves, three-dimensional layered half space, the method of fundamental solution (MFS), alluvial basin.
\end{abstract}

\section{Introduction}

The scattering of elastic waves is a hot topic in many fields, which are widely used in earthquake (blast) wave analysis, geophysical prospecting, nondestructive testing and so on. Generally speaking, theoretical analysis methods can be divided into analytical method and numerical method, and the later includes the domain discrete numerical methods such as the finite element method, finite difference method and the boundary- discrete methods. While the boundary-discrete methods include the method of fundamental solution (MFS), the discrete wave number method, the boundary element method and other similar methods. Among them, the MFS, also referred to superposition method, general inverse method, the virtual boundary element method or wave source 
method, is essentially a special indirect boundary integral equation method. The MFS solves wave motions in the following way: virtual wave sources, by which scattered waves are constructed, are placed close to the boundaries of scatterers, and magnitudes of the virtual wave sources are determined by the boundary conditions. Compared with the BEM, this method has several advantages such as avoiding dealing with the singularity of the fundamental solution, and the meshless feature. Due to its excellent numerical accuracy and ease of implementing, it has been widely applied in the field of diffraction of elastic waves in elastic medium [1-4]. More related papers can be found in the survey articles [5-6].

In the field of civil and earthquake engineering, the horizontal multi-layered half space is a reasonable and convenient approximation of the earth in reality. For solving the scattering of elastic waves in layered half space, the MFS is implemented based on dynamic Green's function in layered half space. Compared to the whole-space Green's function, this Green's function is complicated in calculation, but avoids the discretization of the free surface and layer interfaces, which can greatly reduce the equation size and the computational storage. Based on the MFS, the procedure for solving elastic waves scattering in layered half space is presented in this paper. Then, the proposed method is utilized to solve the scattering of elastic waves by the threedimensional basin, in layered medium, and finally some important conclusions are obtained.

\section{The MFS for three-dimensional scattering in layered half space}

As shown in Figure 1, the scatterers such as basin, inclusion are contained in layered half space. Assuming each layer is filled with a homogeneous and isotropic medium, and plane waves incident from the bedrock half space. Firstly, the total wave field is divided into the free field and the scattered waves field. Then according to the single layer potential theory, scattered waves are

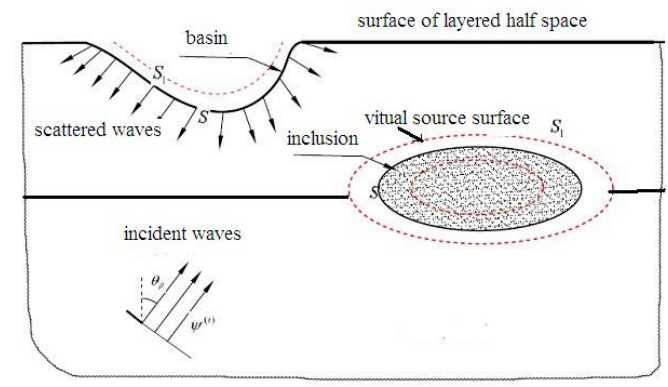

Figure 1: Scattering of elastic waves by heterogeneous body in layered half space. 
constructed by virtual wave sources placed close to the boundary of the scatterer, and the displacements and stresses of the scattered wave field can be expressed as

$$
\begin{array}{cc}
u_{i}(\mathrm{x})=\int_{S} g_{u, i j}(\mathrm{x}, \xi) \phi_{j}(\xi) d s_{\xi} & (i, j=x, y, z) \\
t_{i}(\mathrm{x})=\int_{S} g_{t, i j}(\mathrm{x}, \xi) \phi_{j}(\xi) d s_{\xi} & (i, j=x, y, z)
\end{array}
$$

where $S$ and $S^{\prime}$ are the boundary of the scatterer and the virtual sources surface, respectively; $\mathrm{x} \in S, \xi \in S^{\prime} ; \phi_{j}(\xi)$ are amplitudes for the virtual force acted in $j$ direction located at $\xi \cdot g_{u, i j}(\mathrm{x}, \xi)$ and $g_{t, i j}(\mathrm{x}, \xi)$ are the Green's functions for displacement and traction in $i$ direction due to the application of a unit force in $j$ direction at point $\xi$ respectively in layered half space.

For the actual problem, the above integral equations should be solved by numerical discretization according to the free or continue boundary conditions of the problem. Note that for the scattered waves in inclusion or sedimentary basin, virtual forces should be placed outside the scatterers. For convenience, the shape of virtual source surface is assumed identical to that of the boundary of the scatterers.

Generally speaking, singularity appears if wave sources are acted on the scatterer surface. In this study, the singularity is avoided as the wave sources are located at the virtual surface $S_{1}$ (close to but not on the boundary $S$ ). However, as one disadvantage of MFS, the auxiliary surface needs to be placed at appropriate location, and the optimal distance between the auxiliary surface and the real boundary surface mainly depends on the incident frequency and is usually determined by numerical experience. Numerical experiments show that for incident frequencies $\eta \leq 2.0$ ( $\eta$ is the dimensionless frequency defined later) the wave sources should better be placed at the positions within 0.4-0.6 times of the relevant radii of the scatterer, and the number of the wave sources can approximately take half of that of discrete points of cavity boundary; for higher incident frequency $\eta>2.0$ the wave sources should be placed within $0.7-0.9$ times of the relevant radii of the scatterer, and the number of the wave sources can approximately take 0.8 times of that of discrete points of boundary. While considering the dynamic stress concentration in the corner of some kind of scatterers, the local refinement is suggested to obtain more accurate stress results.

The elastic wave scattering in three dimensional layered half space can be solved with the MFS by utilizing the following steps:

(1) the boundary points collocation: due to the dynamic Green's function for layered half space employed as the fundamental solution, there is no need to discretize the free surface and layer interfaces, the collocation points are only required at the scatterer surfaces.

(2) the free field calculation: in the absence of scatterers, the dynamic response of layered half space should be solved under the incident waves, and the surface 
displacement and stress response on collocation points at scatterer boundary should be calculate for the equation construction.

(3) the scattered wave field construction: virtual source surface is placed near the scatterer surface, and the dynamic response in the medium can be constructed by superposition of all the virtual forces distributed on the virtual source surface. This process is known as dynamic Green function calculation.

(4) boundary equation solution and wave fields superposition: based on the result of above steps, according to the boundary conditions, numerical equation can be constructed and solved. Once the virtual forces density is obtained, the total field response can be obtained by superposition of free field and the scatted wave field.

\subsection{Calculation of Green's function for layered half space}

Consider a concentrated force embedded in layered half space, the dynamic response at arbitrary position can be solved by the method of direct stiffness matrix, which needs to introduce virtual surface on the loading surface, then the loaded layer is divided into two sub-layers. When the MFS or other boundary integral methods is used to solve practical problems, the Green functions of a large number of force sources at different locations are needed, then the stiffness matrix calculation should be updated in every time calculation of green functions, making the processing more complicated and adding large amount of calculation. In addition, when the load and the receiving points are at the same or close horizons, due to the high oscillation of Bessel function, integral function converges quite slowly. Therefore, a modified stiffness matrix method is proposed herein to overcome these problems, which makes the progress very convenient, in the meanwhile it will greatly reduce the amount of calculation in boundary element calculation for three-dimensional wave motion in layered half space.

The process of the green function calculation is shown in figure 2. Firstly, by means of Hankel transform and the Fourier transform in wave number domain, the dynamic stiffness matrix of each layer is calculated, then the global stiffness matrix is obtained by assembling each layer stiffness matrix, which is similar to the finite element method, and the difference only lies in that the layer stiffness matrix is derived analytically and is completely accurate. Secondly, the up and bottom surfaces of loaded layer are fixed, then the reaction forces at two "fixed ends" should be calculated in the wave number domain (fig. 2(b)). The reaction forces can be obtained by superposition of the particular solution and homogeneous solution. Thirdly, the reaction forces at two "fixed ends" are relaxed, then the direct stiffness matrix method is employed to obtain the displacement at layer interfaces (fig. 2(c)). The dynamic response within each layer can be obtained through the transformation matrix. The dynamic response within the loaded layer should be obtained by additional superposition of the "fixed layer" solution. The detailed solving process is shown in paper [7]. 


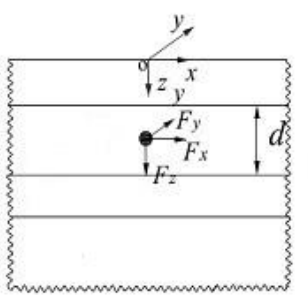

(a)

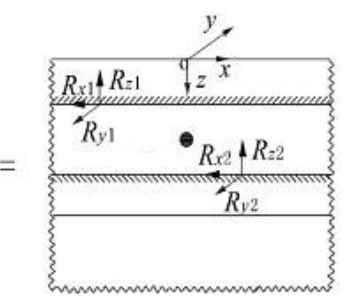

(b)

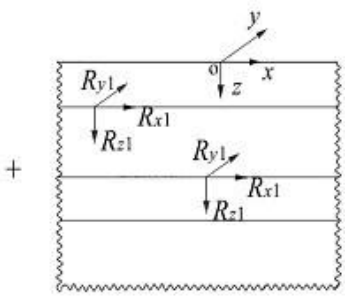

(c)

Figure 2: The solution of green function of harmonic concentrated force source by direct stiffness method

\subsection{Solution}

Because the Green's functions for layered half space are employed, the boundary conditions at the surface of the half-space and layer interfaces have already been satisfied, and only the boundary conditions at the surface of the scatterer need to be considered. Taking the 3-D alluvial basin as an example, to obtain the numerical solution, define the number of the collocation points on boundary and that of the wave sources inside the basin are $N$ and $N_{1}\left(N \geq N_{1}\right)$ respectively, then the displacement and stress of the scattered field outside the basin can be written as

$$
\begin{gathered}
u_{i}^{\mathrm{s}}\left(x_{n}\right)=\sum_{\mathrm{n} 1=1}^{\mathrm{N} 1} b_{n 1} G_{i, 1}^{(s)}\left(x_{n}, x_{n 1}\right)+c_{n 1} G_{i, 2}^{(s)}\left(x_{n}, x_{n 1}\right)+d_{n 1} G_{i, 3}^{(s)}\left(x_{n}, x_{n 1}\right) \\
\sigma_{i j}^{\mathrm{s}}\left(x_{n}\right)=\sum_{\mathrm{n} 1=1}^{\mathrm{N} 1} b_{n 1} T_{i j, 1}^{(s)}\left(x_{n}, x_{n 1}\right)+c_{n 1} T_{i j, 2}^{(s)}\left(x_{n}, x_{n 1}\right)+d_{n 1} T_{i j, 3}^{(s)}\left(x_{n}, x_{n 1}\right) \\
\quad x_{n} \in S, x_{n 1} \in S_{1} ; \quad n=1, \cdots, N ; n 1=1, \cdots, N_{1} ;
\end{gathered}
$$

In which, $b_{n 1}, c_{n 1}$ and $d_{n 1}$ are the amplitude of the concentrate forces acted in $\mathrm{x}, \mathrm{y}$ and $\mathrm{z}$ directions at $n 1$ th source, respectively.

As for the scattered wave field inside the alluvial basin, the virtual source surface $S_{2}$ should be located outside the basin and can be discretized in the same manner. Then according to the continue boundary conditions at the interface between the alluvial basin and half space, the following equations can be obtained

$$
H_{1} Y_{1}+F=H_{2} Y_{2}
$$

In which, $H_{1}\left(6 N, 3 N_{1}\right), H_{2}\left(6 N, 3 N_{2}\right)$ are influence matrix which correspond to the displacement and stress due to virtual wave sources on surfaces $S_{1}$ and $S_{2}$, respectively. $Y_{1}\left(3 N_{1}, 1\right), Y_{2}\left(3 N_{2}, 1\right)$ are unknown density vector. $F(3 N, 1)$ is the displacement and stress vector of the free field. The equation can be solved by using least-square method or pseudo-inverse method. Once the unknown wave source density is determined, the whole wave field will be obtained through the superposition of the scattered wave field and the free field. 


\section{Verifications}

To verify the numerical precision of the method, figure 3 shows the surface displacement amplitudes around a hemisphere basin with radius $a$ in homogeneous half-space compared with the results of Mossessian and Dravinski [3]. The parameters for the half-space are as follows: Poisson's ratio $1 / 3$, damping ratio 0.005 , dimensionless frequency $\eta=\omega a / \pi c_{s}=0.5$, oblique incident plane $\mathrm{P}$ and SV waves with $\theta_{\alpha}\left(\theta_{\beta}\right)=60^{\circ}$. It is shown that the results of the present study agree well with those of Mossessian and Dravinski [3].

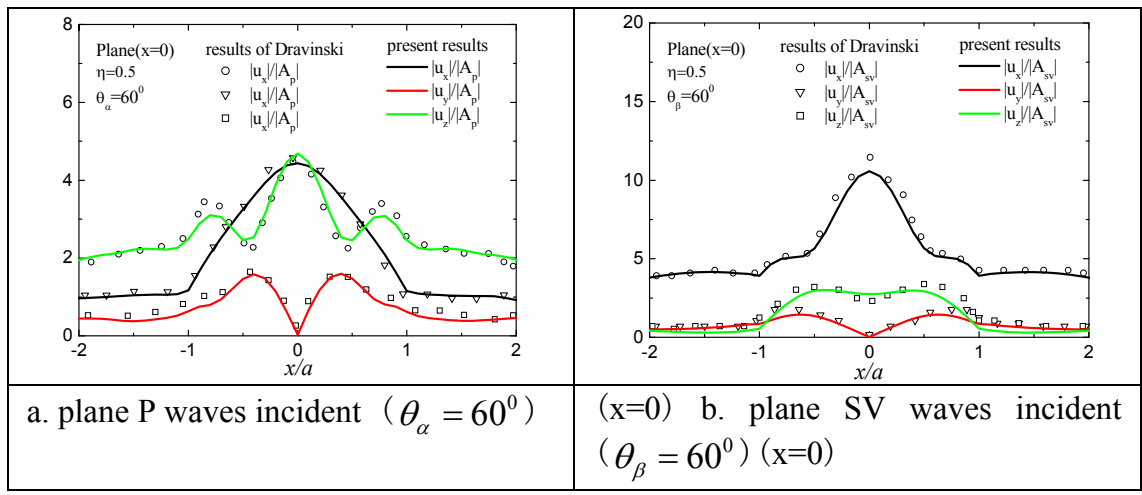

Figure 3: The surface displacement amplitudes of a hemisphere alluvial basin in homogeneous half-space for P and SV waves incident compared with the results of Mossessian and Dravinski.

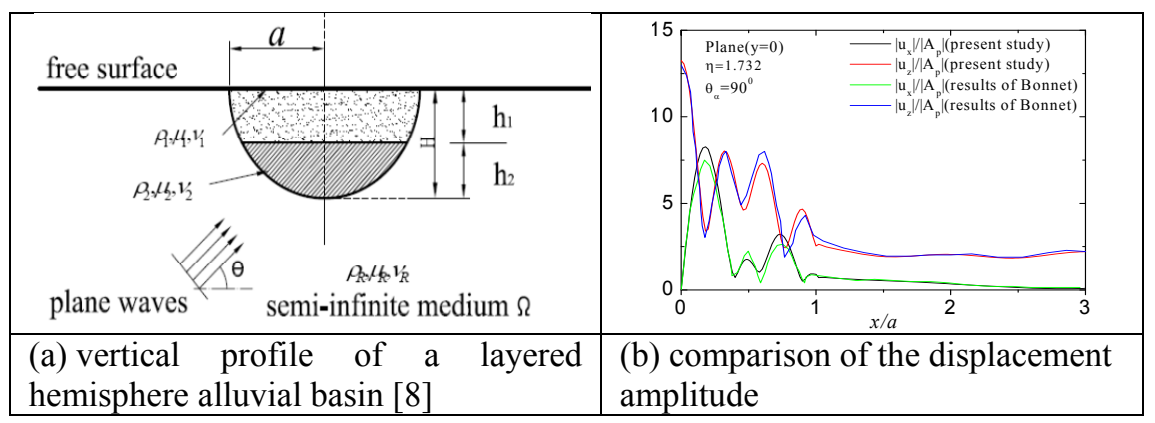

Figure 4: The surface displacement amplitudes of a layered hemisphere alluvial basin for $\mathrm{P}$ waves incident compared with the results of Chailat et al. [8].

For a layered medium, figure 4 shows the surface displacement amplitudes of a hemisphere layered basin with radius $a$ in half-space compared with the results in Chaillat et al. [8]. The parameters for the half-space are as follows: 
Poisson's ratio $v_{R}=0.25, \quad v_{2}=0.3, \quad v_{1}=0.34$, damping ratio 0.001 dimensionless frequency $\eta=\omega a / \pi c_{s}=1.732$ density ratio $\rho_{1} / \rho_{2}=\rho_{2} / \rho_{R}=$ 0.6 , shear modular ratio $\mu_{1} / \mu_{2}=\mu_{2} / \mu_{R}=0.3$, alluvial layer thickness $h_{1} / H=0.586$, vertical incidence of plane $\mathrm{P}$ and SV waves. It is shown that the results of the present study agree well with those of Chaillat et al. [8]. Note that the green function for whole space is used by them, requiring the elements discretization on layer interfaces and the free surface, which has a high demand on the storage capacity and computing ability of the computer for multi-layered medium.

\section{Numerical example}

In this part, the MFS is applied to study the scattering of seismic waves in a layered alluvial basin, and some important scattering phenomena of this model are discussed. Figures 5 and 6 illustrate the surface displacement amplitude
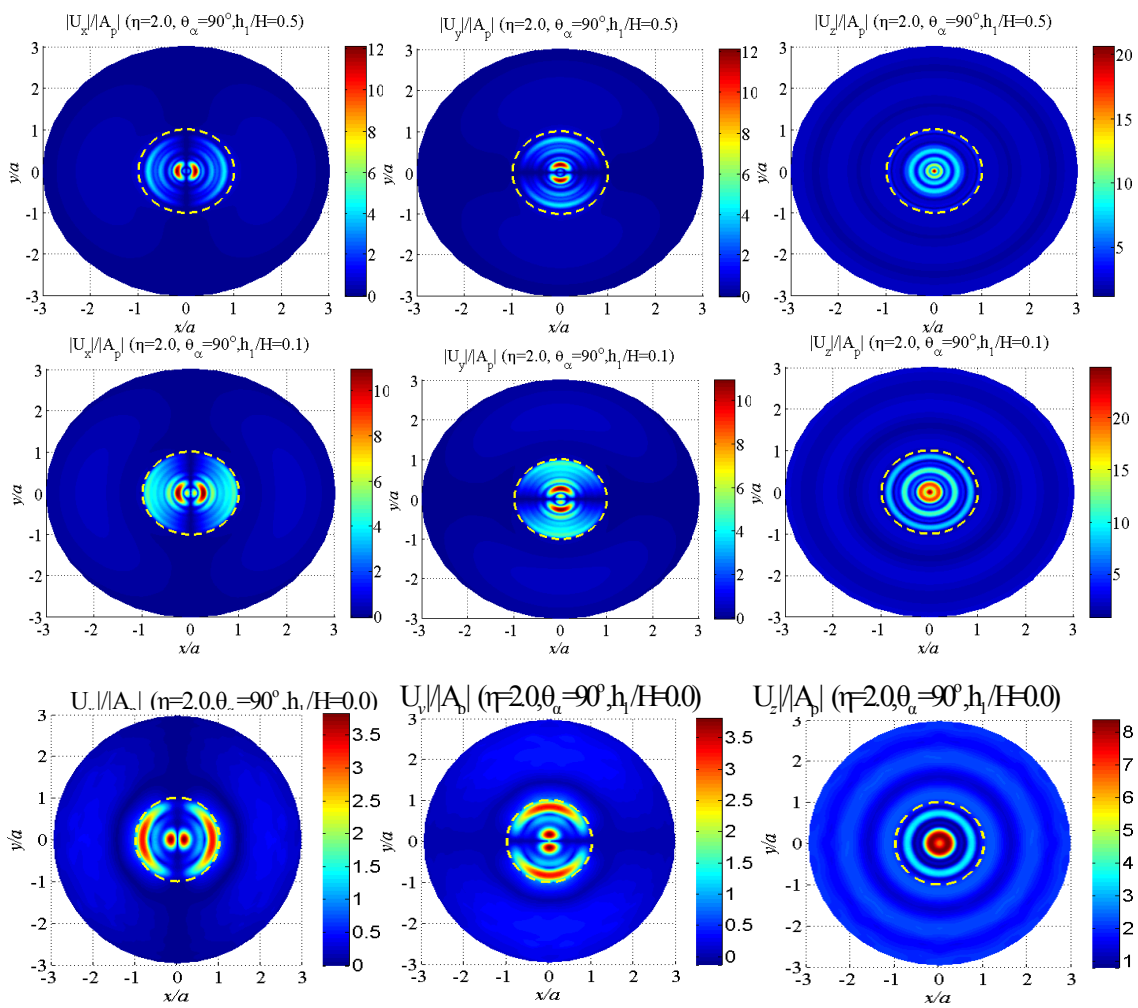

Figure 5: The contour of displacement amplitude around a layered hemispherical alluvial basin for $\mathrm{P}$ waves incident $\left(\mathrm{h}_{1} / \mathrm{H}=0.5,0.1\right.$, $0.0, \quad \eta=2.0)$. 
around a hemisphere two-layered basin with radius $a$ embedded in half-space. The parameters are as follows: Poisson's ratio $v_{R}=0.25, v_{2}=0.3, v_{1}=0.34$, damping ratio 0.001 , density ratio $\rho_{1} / \rho_{2}=\rho_{2} / \rho_{R}=2 / 3$, shear modular ratio $\mu_{1} / \mu_{2}=\mu_{2} / \mu_{R}=1 / 6$, the ratio between the thickness of the upper layer and that of the total depth of the basin $\mathrm{h}_{1} / \mathrm{H}=0.5,0.1,0.0$, dimensionless frequency $\eta=$ $\omega a / \pi c_{s}=2.0$, incident angle $\theta_{\alpha}\left(\theta_{\beta}\right)=90^{\circ}$.
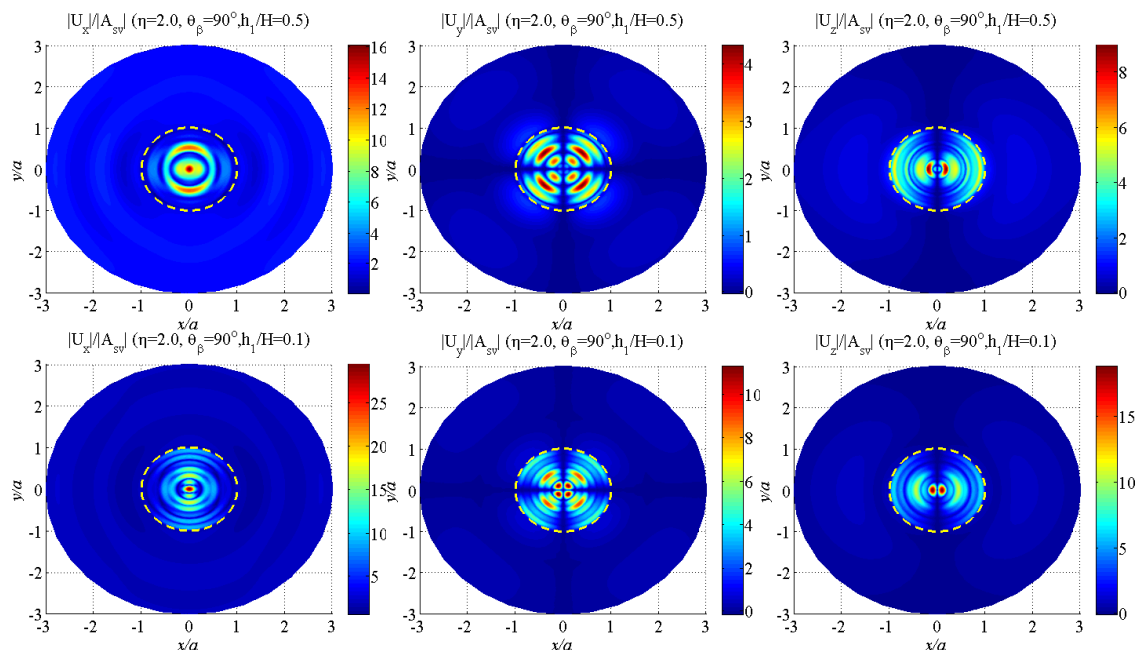

$\left|\mathrm{U}_{\mathrm{y}}\right| / \mathrm{A}_{\mathrm{SW}}\left(\boldsymbol{\eta}=2.0, \theta_{\beta}=90^{\circ}, \mathrm{h}_{1} / \mathrm{H}=0.1\right)$

$\left|\mathrm{U}_{z} / / \mathrm{A}_{\mathrm{sv}}\right|\left(\eta=2.0, \theta_{\mathrm{g}}^{\mathrm{x} / a}=90^{\circ}, \mathrm{h}_{7} / \mathrm{H}=0.1\right)$
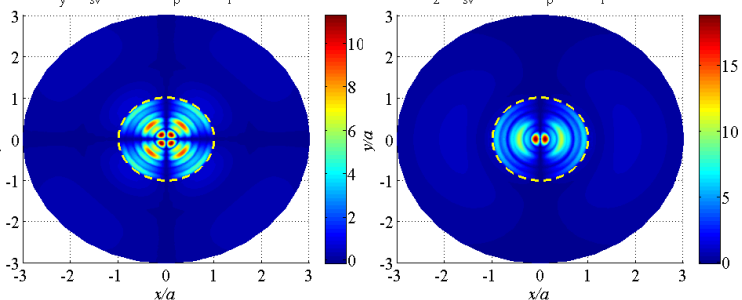

$\mathrm{U} / / \mathrm{A} \mid\left(\mathrm{\eta}=2.0, \theta_{\alpha}=90^{\circ}, \mathrm{h}_{1} / \mathrm{H}=0.0\right)$

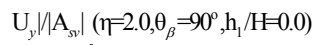

$\mathrm{U}_{z} / /\left|\mathrm{A}_{s v}\right|\left(\eta=2.0, \theta_{\beta}=90^{\circ}, \mathrm{h}_{1} / \mathrm{H}=0.0\right)$
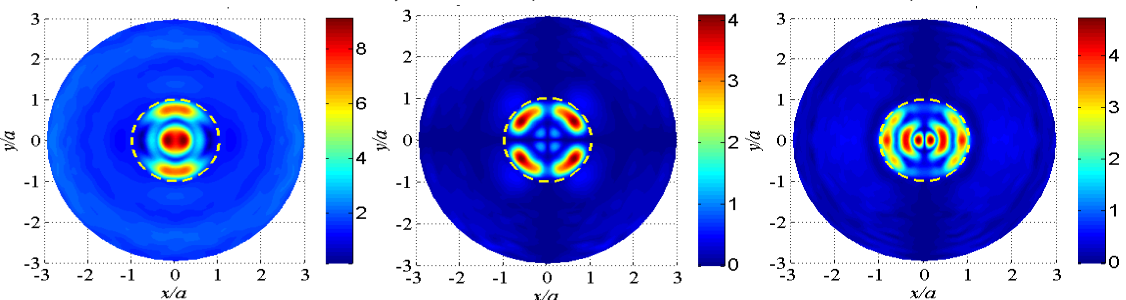

Figure 6: The contour of displacement amplitude around a layered hemispherical alluvial basin for $\mathrm{SV}$ waves incident $\left(\mathrm{h}_{1} / \mathrm{H}=0.5,0.1\right.$, $0.0, \quad \eta=2.0)$.

From these figures, significant amplification effects can be seen clearly both in the centre and the edge of basin due to the focusing of seismic waves and the surface wave transformation. Consider the change of the thickness of the upper layer, it is shown that there is a large difference between the scattering phenomenon of the two-layered basin and the homogenous basin $\left(h_{1} / H=0.0\right)$, and the amplification effect seems more pronounced, and the displacement amplitude oscillates more rapidly in space in the former case. For example, the maximum displacement amplitudes of the layered basin reach 18.7 and 36.1 for 
$\mathrm{P}$ and SV waves incident vertically respectively, about 2.3 and 3.5 times that of the homogenous basin. It may be explained that the seismic waves are multiplereflected between the upper and bottom surface of the near surface soft layer and seismic energy are mainly trapped in this layer. Correspondingly, the thickness of the upper layer greatly influences the reflection and superposition of waves. Hence, the accurate modelling of the seismic response of alluvial basin requires more detailed geological exploration data.

\section{Conclusions}

(1) This paper presents the method of fundamental solution (MFS) for solving the scattering of elastic waves around three-dimensional scatterers, based on dynamic Green's function in layered half space. It is illustrated that the threedimensional wave motion in layered medium can be efficiently and accurately solved by the proposed method. In addition, the MFS is a meshless method, which only needs a few of collocation points on the scatterer surface, so it can be implemented fairly conveniently.

(2) Numerical results illustrate that the scattering of seismic waves by threedimensional layered sedimentary basin is significantly different from that of the homogenous basin, and the wave energy can be largely trapped in the near surface soft layer. It is necessary to consider the bedding structure of the alluvial basin for seismic wave modelling in reality.

\section{Acknowledgement}

The authors gratefully acknowledge the support from National Natural Science Foundation of China under grants 51008210 and 51278327.

\section{References}

[1] Wong H L. Effect of surface topography on the diffraction of P, SV, and Rayleigh waves. Bulletin of the Seismological Society of America, 72, pp. 1167-1183, 1982.

[2] Dravinski M. Scattering of waves by a sedimentary basin with a corrugated interface. Bulletin of the Seismological Society of America, 97(1B), pp. 256264, 2007.

[3] Mossessian T K., Dravinski M. Amplification of elastic waves by a three dimensional valley, Part 1: steady state response, Earthquake Engineering and Structural Dynamics, 19, pp. 667-680, 1990.

[4] Antonio Tadeu, Lu Godinho, Julieta Antonio, et al. Wave propagation in cracked elastic slabs and half-space domains - TBEM and MFS approaches. Engineering Analysis with Boundary Elements, 31, pp. 819-835, 2007.

[5] Golberg, M. A.; Chen, C. S.: The method of fundamental solutions for potential, Helmholtz and diffusion problems. Boundary Integral Methods: Numerical and Mathematical Aspects, Ed. M.A. Golberg, WIT Press and 
Computational Mechanics Publications, Boston, Southampton, pp. 103-176, 1998.

[6] Graeme Fairweather, Andreas Karageorghis, P.A. Martin. The method of fundamental solutions for scattering and radiation problems. Engineering Analysis with Boundary Elements, 27, pp. 759-769, 2003.

[7] Zhongxian Liu, Jianwen Liang. Dynamic Green's function for threedimensional concentrated loads in the interior of viscoelastic layered halfspace - Modified Stiffness Matrix Method (in Chinese). Acta Mechanica Solida Sinica (accept)

[8] Chaillat S., Bonnet M. and Semblat J F. A new fast multi-domain BEM to model seismic wave propagation and amplification in 3-D geological structures, Geophysical Journal International, 177, pp. 509-531, 2009. 